\title{
sciendo
}

\author{
BULGARIAN ACADEMY OF SCIENCES
}

CYBERNETICS AND INFORMATION TECHNOLOGIES • Volume 20, No 6

Special Issue on New Developments in Scalable Computing

Sofia $\bullet 2020$

Print ISSN: 1311-9702; Online ISSN: 1314-4081

DOI: $10.2478 /$ cait-2020-0069

\section{Trend Analysis of CMIP5 Ensemble of Climate Indices over Southeast Europe with Focus on Agricultural Impacts}

\author{
Hristo Chervenkov ${ }^{1}$, Georgi Gadzhev ${ }^{2}$, Vladimir Ivanov², Kostadin \\ Ganev $^{2}$ \\ ${ }^{1}$ National Institute of Meteorology and Hydrology (NIMH), Tsarigradsko Shose Blvd. 66, 1784 Sofia, \\ Bulgaria \\ ${ }^{2}$ National Institute of Geophysics, Geodesy and Geography - Bulgarian Academy of Sciences (NIGGG- \\ BAS), Acad. Georgi Bonchev Str. Bl. 3, 1113 Sofia, Bulgaria \\ E-mails: hristo.tchervenkov@meteo.bgvivanov@geophys.bas.bgｇgadzhev@geophys.bas.bg \\ kganev@geophys.bas.bg
}

\begin{abstract}
Nowadays there is a strong degree of agreement that the climate change is the defining challenge of our time, which will exert influence on the ecosystems, on all branches of the international economy and on the quality of life. The analysis based on climate indices is widely used non-parametric approach for quantification of the mean state as well as extreme climate events. This study, which is continuation of our previous efforts, is dedicated to the assessment of the trend magnitude and the trend statistical significance of six temperature-based and three precipitation-based indices in projected future climate over Southeast Europe up to the end of the 21st century. The indices are computed from the bias-corrected output of five CMIP5 global models, reinforced with all four RCP emission scenarios. The model output is accessed from the section of the Inter Sectoral Impact Model Intercomparison Project in the Copernicus Data Store. The multi model ensemble medians of the temperature-based indices shows considerable increase which is consistent with the warming of the mean temperatures. These changes are statistically significant in most cases and intensify with the radiative forcing. The revealed tendencies of the precipitation-based indices are more complex when compared with temperature tendencies.
\end{abstract}

Keywords: Climate indices, RCP, CMIP5 Ensemble, future climate, trend analysis.

\section{Introduction}

Nowadays there is a strong degree of agreement that the climate change is the defining challenge of our time, which will exert influence on the ecosystems, on all branches of the international economy and on the quality of life. However, as stated in [18], immediate damages to humans and ecosystems are not caused by gradual changes in the mean state but mainly by so-called extreme climate events. The 
extreme weather phenomena are discussed in all reports of the Intergovernmental Panel on Climate Change. There are various methods to investigate extreme events, but the computation and analysis of Climate Indices (CIs), derived from daily data (observations or model output) is probably the most widely used non-parametric approach. Subsequently, the literature is very large ([1-3, 18, 19] and many others). To detect changes in these extremes, it is important to apply sets of CIs that are statistically robust, cover a wide range of climate conditions, and have a high signalto-noise ratio [1]. Such sets are used in several projects on climate change with a focus on different spatial scales and temporal extent.

The relevance of the trend assessment of climate extremes is frequently emphasized (see, for example, $[1,18]$ and references therein). The principal reason is that it is vital to discover the presence of long-term persistent tendencies as well as to quantify their magnitude.

Our working group has previous, partially project-driven, experience with CIsbased analysis [4-6]. The present study is a natural continuation of our recent efforts, documented in $[7,8]$. These two studies are dedicated to the assessment of the future climate over SouthEast (SE) Europe, and are based on an ensemble of CIs with the main goal to compare the multiyear CIs-means for the present and the projected future climate. The main aim of the present work is to assess the trend magnitude and statistical significance of six temperature-based and three precipitation-based indices in projected future climate over the same domain up to the end of the 21st century in a consistent manner. The indices are computed from the bias-corrected output of five CMIP5 global models, reinforced with all four RCP emission scenarios. As in [18] and [19] and many other similar studies, the present one exploits the ensemble multimodel median, noted henceforth MMX50, rather than the output from the individual models.

The article is structured as follows: The used scenarios, models, and input data are concisely described in the second section. The theoretical background of the performed statistical analysis is placed in the third section. The core of the article is in the fourth section where the performed computations and the obtained results are described. The brief concluding remarks are in the last section.

\section{Scenarios, models and input data}

The Coupled Model Intercomparison Project (CMIP) is a standard experimental protocol for studying the output of Coupled Atmosphere-Ocean General Circulation Models (CAOGCMs). The main aim of the fifth phase of CMIP, CMIP5, is to study the climate and climate change in the past, present, and future, using a set of simulations with different climate simulators in various spatial and temporal scales [22]. The CMIP5 experiment uses a set of emission scenarios called Representative Concentration Pathways (RCP) [15] to assess the interactions between the human activities on the one hand and the environment on the other hand, and their evolution. In contrast to the previous generations of scenarios, the RCPs are mitigation scenarios that assume active policy actions directed to achieve certain emission targets. Four RCPs have been formulated: RCP2.6, RCP4.5, RCP6.0 and RCP8.5. They are based 
on a range of projections of future population growth, technological development, and societal responses. The labels for the RCPs provide a rough estimate of the radiative forcing in the year 2100 (relative to pre-industrial conditions).

The present study exploits the database whose creation is described in [7], whereas the primary data access point was the Copernicus Data Store. It is based on the collection of bias-corrected climate datasets provided through Inter Sectoral Impact Model Intercomparison Project (ISIMIP 1, https://www.isimip.org/protocol/), Fast Track simulation round. These climate datasets contain daily-resolution, biascorrected climate data from five CMIP5 CAOGCMs according to Table 1 covering the period 1950-2099 (historical run - up to 2005 and CMIP5 simulations for 20112099), downscaled to a $0.5^{\circ} \times 0.5^{\circ}$ lat-lon grid. The main goal of ISIMIP is to offer reliable global climatological data for agro-climatic impact assessments but most of the included variables have universal applicability.

Table 1. CAOGCMs used in ISIMIP 1 Fast Track simulation round
\begin{tabular}{|c|c|c|}
\hline Model Acronym & Institution, country & Spat. Res. (Lon. $\times$ Lat. $\times$ Lev.) \\
\hline GFDL-ESM2M & Geophysical Fluid Dynamics Laboratory, USA & $144 \times 90 \times 24$ \\
\hline HadGEM2-ES & Met Office Hadley Centre, UK & $192 \times 145 \times 40$ \\
\hline IPSL-CM5A-LR & Institut Pierre-Simon Laplace, France & $96 \times 96 \times 39$ \\
\hline MIROC-ESM-CHEM & AORI, NIES, JAMSTEC, Japan & $128 \times 64 \times 80$ \\
\hline NorESM1-M & Norwegian Climate Centre, Norway & $144 \times 96 \times 26$ \\
\hline
\end{tabular}

As emphasized in the introduction, the study is based entirely on the MMX50.

The selected CIs are the same as in [7] and [8] where the six temperature-based are: Minimum of the Minimum Temperatures, Maximum of the Maximum Temperatures, Frost Days, Tropical Nights, Cold and Warm Spell Duration Indices, noted traditionally as TNn, TXx, FD, TR, CSDI and WSDI. The three precipitation based indices are Annual Precipitation Sum, Heavy Precipitation Days and Consecutive Dry Days, noted RR, RR10mm and CDD, respectively. In the articles above-referenced, the reader could find the explanatory motivation of this selection as well as links to the definitions of these CIs.

\section{Methods}

The magnitude of the trend is estimated by means of the Theil-Sen Estimator (TSE). The method TSE is named after H. Theil and P. Sen, who published the pioneering papers ([12] and [17]). Conceptually, the method is very simple: If $x_{i}, i=1, \ldots, n$, and $y_{i}, i=1, \ldots, n$, are the independent (most frequently the time) and dependent variables correspondingly, the estimation of the model is done by calculating the slopes of all possible $\left(\begin{array}{l}n \\ 2\end{array}\right)=\frac{n(n-1)}{2}$ combinations of pairs of points. The slope between every two points is equal to

$$
\beta=\frac{y_{j}-y_{i}}{x_{j}-x_{i}}, x_{i} \neq x_{j}
$$


The final non-parametric slope $\hat{\beta}_{1}$ is then defined as the spatial median of these slopes:

$$
\hat{\beta}_{1}=\operatorname{median}\{\tilde{B}\},\left\{\tilde{B}=b_{i j} \mid b_{i j}=\frac{y_{j}-y_{i}}{x_{j}-x_{i}}, x_{i} \neq x_{j}, 1 \leq i<j \leq n\right\} .
$$

Positive value of $\hat{\beta}_{1}$ reveals an increasing trend, negative value is sign of decreasing trend.

The TSE is essentially an estimator for the slope alone; the line has been constructed by means of different methods. In fact there are a large variety of ways to calculate the intercept $\hat{\beta}_{0}$. We apply the frequently used relation, proposed in [9]:

$$
\hat{\beta}_{0}=Y_{\text {median }}-\hat{\beta}_{1} X_{\text {median }} \text {, }
$$

where $Y_{\text {median }}$ and $X_{\text {median }}$ are the medians of the dependent and independent variables correspondingly.

The main strength of the TSE is its robustness. As stated by many authors (see [10] and references therein), the TSE, compared to the frequently used ordinary Least Squares Estimation (LSE), is much less sensitive to outliers. This conclusion could be explained intuitively so: A positive outlier will increase the sample mean in direct proportion to the size of the outlier. In fact, there is no upper limit on the effect that can be induced on the sample mean by an outlier. On the other hand, the effect of an outlier on the sample median is bounded; once the outlier becomes the largest observation in the sample it has no further influence on the median [20]. In fact, the breakdown point of TSE is $1-1 / \sqrt{2} \approx 29.3 \%$, meaning that it can tolerate arbitrary corruption of up to $29.3 \%$ of the input data-points without degradation of its accuracy [23]. The performance of both regression methods, LSE and TSE, for trend estimation of meteorological data is assessed in [4]. Among other findings, it is revealed that in certain cases the LSE could produce trend slope even with wrong sign. The TSE produces for the same dataset reliable result.

Although $O\left(n^{2}\right)$ procedure, the TSE is still computationally feasible, keeping in mind that in the most geophysical and engineering applications $n<1000$.

The statistical significance of the trend is estimated by means of the MannKendall (MK) test [12, 13].

Like the TSE, the MK is non-parametric rank-based procedure. The null and the alternative hypothesis of the two-sided test, denoted traditionally $H_{0}$ and $H_{A}$ of the MK test for trend in the random variable $x$ are as follows:

$$
\begin{array}{ll}
H_{0}: \quad \operatorname{Pr}\left(y_{j}>y_{i}\right)=0.5, \quad j>i, \\
H_{A}: \quad \operatorname{Pr}\left(y_{j}<y_{i}\right) \neq 0.5, \quad j>i .
\end{array}
$$

The MK statistic $S$ is calculated as

$$
S=\sum_{k=1}^{n-1} \sum_{j=k+1}^{n} \operatorname{sgn}\left(y_{j}-y_{k}\right), \quad j>k,
$$

where $y_{i}$ and $y_{k}$ are the values of the considered variable in the moments $j$ and $k$, respectively, $n$ is the total number of years and the sign function is defined as 


$$
\operatorname{sgn}\left(y_{j}-y_{k}\right)=\left\{\begin{array}{l}
1 \text { if } y_{j}-y_{k}>0, \\
0 \text { if } y_{j}-y_{k}=0, \\
-1 \text { if } y_{j}-y_{k}<0 .
\end{array}\right.
$$

For large $n$ (practically for $n \geq 8$ ), the distribution of $S$ can be well approximated by a normal distribution with mean zero and variance computed as

$$
\operatorname{VAR}(S)=\frac{1}{18}\left[n(n-1)(2 n+5)-\sum_{p-1}^{g} t_{p}\left(t_{p}-1\right)\left(2 t_{p}+5\right)\right],
$$

where $g$ is the number of tied groups, and $t_{p}$ is the amount of data with the same value in the group $p=1, \ldots, g$. The normalised test statistic $Z$ is given by:

$$
Z= \begin{cases}\frac{S-1}{\sqrt{\operatorname{VAR}(S)}} & \text { if } S>0, \\ 0 & \text { if } S=0, \\ \frac{S+1}{\sqrt{\operatorname{VAR}(S)}} & \text { if } S<0 .\end{cases}
$$

If the normalised test statistic $Z$ is equal to zero, the data are normally distributed, and the positive values of $Z$ mean a rising trend and negative a decreasing trend [25]. The null hypothesis is rejected at significance level $\alpha$ if $|Z|>Z_{\alpha / 2}$ (two-tail test), where $Z_{\alpha / 2}$ is the value of the standard normal distribution with an exceedance probability $\alpha / 2[3]$.

The probability value ( $p$-value) of the MK test is computed as

$$
p(Z)=0.5-\Phi(|Z|) k d e \Phi(|Z|)=\frac{1}{\sqrt{2 \pi}} \int_{0}^{|Z|} e^{-\frac{t^{2}}{2}} d t .
$$

For a specific significance level $\alpha \in(0,1)$ the null hypothesis is rejected whenever $p(Z)<\alpha$.

Like the TSE, the MK test is a procedure, especially suitable for non-normally distributed data, data containing outliers and nonlinear trends. Consequently, it is widely used in many engineering and geophysical branches as hydrology (see, for example, $[11,25,26])$. They are recommended from the World Meteorological Organisation [24] and are practically standard tools for statistical assessment of trend in the meteorology ([1-6, 17-18] and many others).

\section{Performed computations and obtained results}

All calculations are performed by purposely written from the author's programs in FORTRAN90/95. The parameters of the TSE regression are computed also directly, using (2) and (3). The median slope is calculated exactly by computing all lines through pairs of points. Although quadratic in time, this approach is still feasible due to its simplicity and, as noted above, by the relatively small length of the time series (in case $n=89$, the number of years 2011-2099). The median itself 
is computed with the A. Miller's subroutine (freely-available repository at http://jblevins.org/mirror/amiller/), which implements the efficient truncated quicksort algorithm.

The p-value of the MK-test is also computed directly according to the sequence (5)-(8). The routine, based on Chebyshev fitting [21] is used for the computation of the error function in (9). In the present work, as in many similar studies (e.g., $[3,5,18-19])$, the significance level is fixed at $5 \%$.

Unlike the LSE, benchmarks for the TSE and MK test are difficult to find. In the present study, we use for this goal the representative source [14] and the results are identical.

The magnitudes of the trend in time as well as its statistical significance are estimated individually for all grid cells and separately for each scenario.

Fig. 1 shows the results of the trend analysis for TNn and TXx.

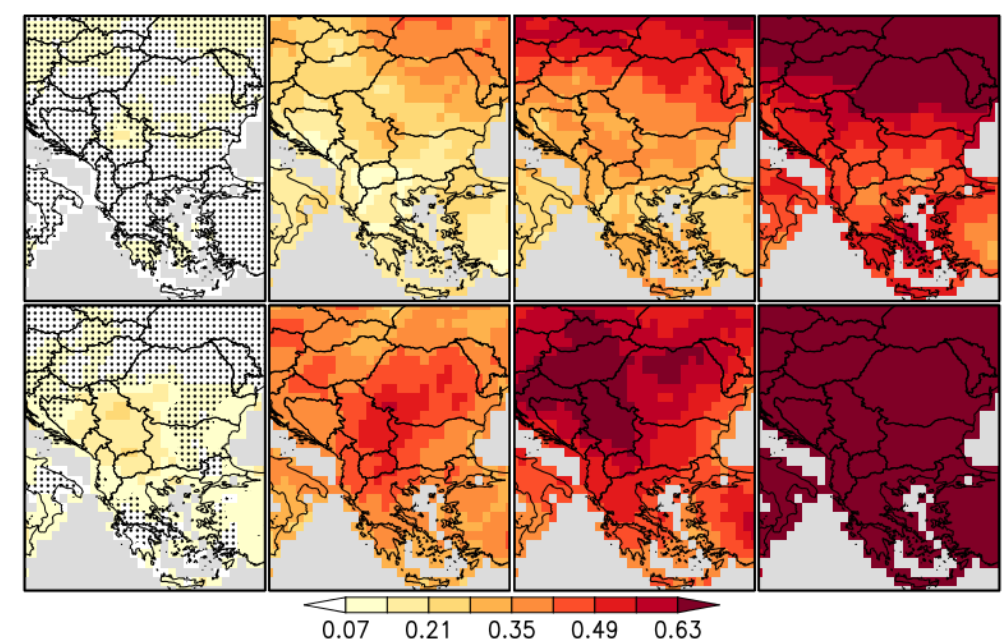

Fig. 1. Trend slopes (unit: ${ }^{\circ} \mathrm{C}$ per 10 years) of the MMX50 of the TNn (first row) and TXx (second row) for the RCP2.6, RCP4.5, RCP6.0 and RCP8.5 in the first, second third and fourth column correspondingly. Stippling indicates grid points with changes that are not significant at the 5\% significance level

Fig. 1 shows a gradual increase of the overall slopes (i.e., the rate of the change, in case increase) of both indices from RCP2.6 to RCP8.5, i.e., proportional to the radiative forcing. The value of the trend magnitude of the TNn appears bigger over the northern part of the domain for RCP6.0 and RCP8.5 but as a whole, the spatial distributions do not shows clear systematic pattern. The most apparent difference between the trend magnitude fields of the TNn and TXx are the generally bigger values for the TXx for all four scenarios. This could be related to the documented in many articles (see, for example, [1]) warming asymmetry. It is worth emphasizing, however, that depending of the considered domain, spatial and temporal scales as well as the methodology applied, the reported results are different, even partially contradicting.

The results for the other temperature based CIs are shown in Fig. 2. 


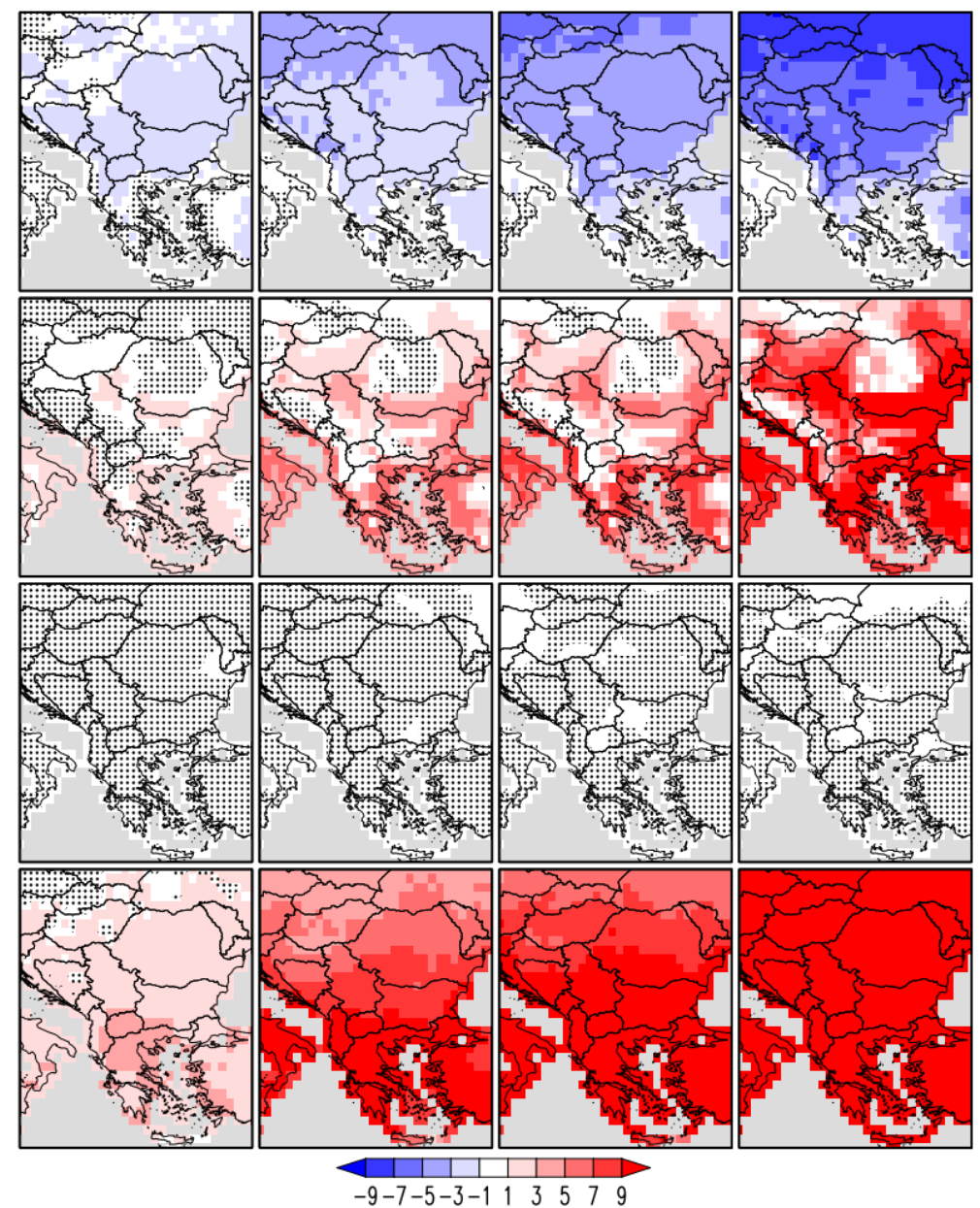

Fig. 2. Same as Fig. 1 but for the FD, TR, CSDI and WSDI on the first second third and fourth row correspondingly. The units are days per 10 years

Consistent with the changes in the mean and extreme temperatures, the overall picture shows progressive (i.e., from RCP2.6 to RCP8.5) decrease of the slope FD and increase of the TR and WSDI. The trends of these CIs are statistically significant over the bigger part of the domain in all scenarios. Somewhat surprising appears the fields of the CSDI: In contrast to the expectations, there is practically no trend under any scenario. The reason is rooted in the relatively small value of this index even in the reference period, as shown in [8]. Thus, under the conditions of the generally warmer climate, the CSDI drops close to zero (i.e., constant), causing the absence of a trend.

The vertical gradient of the trend magnitude is most apparent in the case of the tropical nights especially along the main Carpathian ridge. It is stated in [3] that the occurrence of TR is substantial only in low elevation areas (below $800 \mathrm{~m}$ ), particularly exposed to persistent and intense warm spells in summer. Generally, tropical nights are not characteristic of the mountain climate of the Balkan Peninsula. It is worth to emphasize, however, that the zones with not essential trend increase 
become smaller with the rise of the radiative forcing, suggesting that even the mountains will be exposed in the future to excessive warmth.

As in many other places of the world, in contrast to the projected changes in the temperature indices, where there is a general agreement on the sign of change independent of the region considered, changes in the precipitation indices over the considered region are less consistent in this regard $[8,19]$.

As noted in [8], the considered precipitation-based indices are used as key parameters in many studies of present [18] and projected future climate [19].

Fig. 3 shows the results of the trend analysis for the annual precipitation sum $\mathrm{RR}$ that is probably the most important precipitation-based climate index.

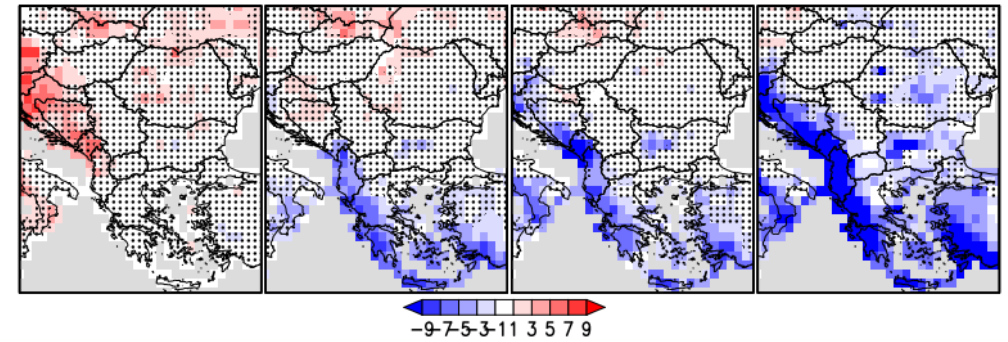

Fig. 3. Same as Fig. 1 but for the RR. The units are mm per 100 years

The most relevant result of the analysis of Fig. 3 is also most noticeable: Except relatively small areas along the Adriatic coast and Asia Minor, there is no statistically significant trend over the domain.

Finally, we present in Fig. 4 the trend analysis outcome for the Heavy Precipitation Days and Consecutive Dry Days.

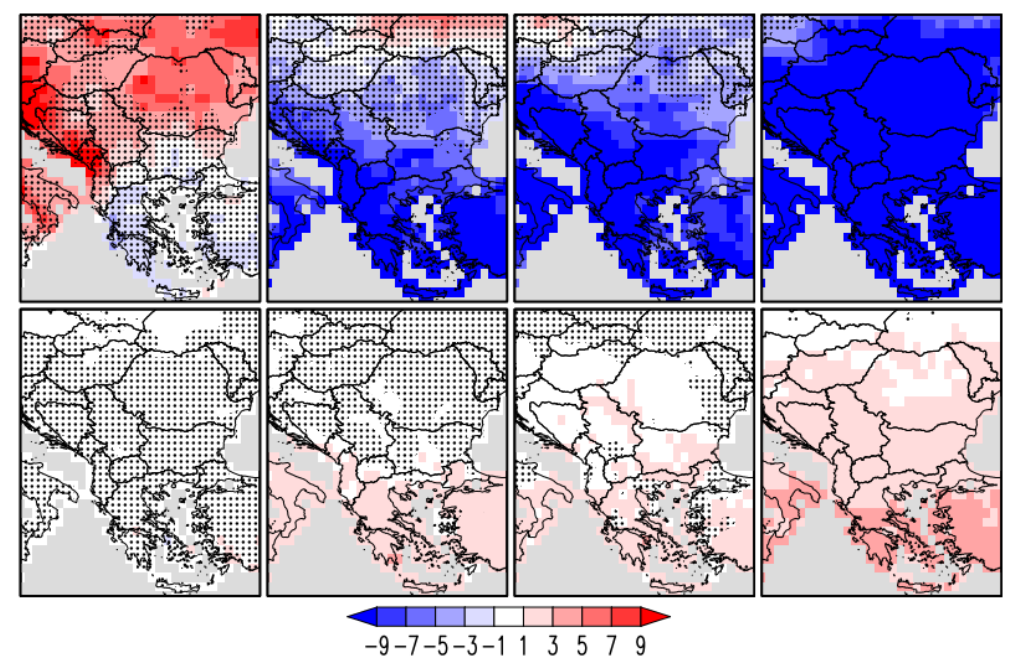

Fig. 4. Same as Fig. 1 but for the RR10 mm and CDD. The units are days per 10 years

The relatively big difference, even in sign, between the trend magnitude for RCP2.6 on one hand and all other three scenarios on the other, is the most notable outcome from the analysis of the RR $10 \mathrm{~mm}$. The trend for RCP2.6 is prevailing 
positive (i.e., increase of the heavy precipitation days) and in the others - dominating negative. It should be kept in mind that RCP2.6 represents mitigation scenarios that aim to limit the increase of global mean temperature to less than $2{ }^{\circ} \mathrm{C}[15]$ and thus it is the only one RCP compatible with the goals of the Paris Agreement. It is worth emphasizing also that the long-term tendencies of some precipitation extremes could deviate from the course of evolution of the mean state [1].

The spatial distribution of the trend magnitude for CDD is consistent in all scenarios. The prevailing trend is positive (i.e., increase of the consecutive dry days) but, as a whole, is relatively weak and, subsequently, statistically significant over the bigger part of the domain only for RCP6.0 and RCP8.5.

\section{Conclusion}

Based on the availability of new sources of information representing the state of the art global climate change simulations in the frame of the CMIP5 project, which are freely accessible from the Copernicus Data Store, we present a trend analysis of key temperature and precipitation-based climate indices over Southeast Europe.

The study confirms the suitability of the database created from ISIMIP1 products for CIs-based analysis of the projected future climate in a computationally feasible way as has been already shown in $[7,8]$. The most important outcome from the study is the notable expressed and spatially dominating positive trend (i.e., uprise tendency) of the warm-related indices and, vice versa, the negative trend of the coldrelated indices. These tendencies are proportional to the radiative forcing and are, as a whole, statistically significant for the scenarios RCP4.5-8.5. The signal of the significant trends is spatially consistent as there are no areas of mixed trends within the considered domain. Generally, the revealed warming is, evidently, a continuation of already detected tendency in the historical records of the twentieth century over the region $[3,5,18]$.

Concerning the precipitation-based indices, the study confirms the complexity of the expected precipitation-related changes and their inherent ambiguity. It is worth emphasizing also that the inter-model spread within the ensemble is bigger compared to the temperature-based CIs and has in some cases systematic character. Most essential in this regard is, however, the revealed absence of trend of the precipitation sum.

Finally, it has to be noticed that the projected increase of the CDD could amplify the negative impact of the expected hotter climate.

\footnotetext{
Acknowledgments: Hence this study is entirely based on free available data and software, the authors would like to express their deep gratitude to the primary CMIP5 model output vendors as well as to all other institutions (MPI-M, UNI-DATA, Copernicus Data Store), which provides free of charge software and data.

This work has been carried out in the framework of the National Science Program "Environmental Protection and Reduction of Risks of Adverse Events and Natural Disasters", approved by the Resolution of the Council of Ministers No 577/17.08.2018 and supported by the Ministry of Education and Science (MES) of Bulgaria (Agreement No Д01-322/18.12.2019).

This work has been accomplished with the financial support by the Grant No BG05M2OP0011.001-0003, financed by the Science and Education for Smart Growth Operational Program (2014-2020) and co-financed by the European Union through the European structural and Investment funds.
} 


\section{References}

1. A l e x a n d e r, L. V., et al. Global Observed Changes in Daily Climate Extremes of Temperature and Precipitation. - J. Geophys. Res., Vol. 111, 2006, D05109. DOI:10.1029/2005JD006290.

2. A le x a nd e r, L. V., J. M. A rbla st e r. Assessing Trends in Observed and Modelled Climate Extremes over Australia in Relation to Future Projections. - Int. J. Climatol., Vol. 29, 2009, pp. 417-435. DOI:10.1002/joc.1730.

3. B i r s a n, M.-V., A. D u m i tre s c u, D. M. M i c u, S. C h e v a l. Changes in Annual Temperature Extremes in the Carpathians Since AD 1961. - Nat. Hazards, Vol. 74, 2014, No 3, pp. 1899-1910. DOI: 10.1007/s11069-014-1290-5. https://doi.org/10.1007/s11069-014-1290-5

4. Chervenkov, H., K. Slavov. Theil-Sen Estimator vs. Ordinary Least Squares - Trend Analysis for Selected ETCCDI Climate Indices. - Compt. Rend. Acad. bulg. Sci., Vol. 72, 2019, No 1, pp. 47-54. DOI:10.7546/CRABS.2019.01.06.

5. C h e r v e $\mathrm{n} \mathrm{k} \mathrm{o} \mathrm{v,} \mathrm{H.,} \mathrm{K.} \mathrm{S} 1$ a v o v. Historical Climate Assessment of Temperature-Based ETCCDI Climate Indices Derived from CMIP5 Simulations. - Compt. Rend. Acad. bulg. Sci., Vol. 73, 2020, No 6, pp. 784-790. DOI: 10.7546/CRABS.2020.06.05.

6. Ch e r v e n k o v, H., K. S l a v o v. Historical Climate Assessment of Precipitation-Based ETCCDI Climate Indices Derived from CMIP5 Simulations. - Compt. Rend. Acad. bulg. Sci., Vol. 73, 2020, No 7, pp. 942-948. DOI:10.7546/CRABS.2020.07.06.

7. Che rve n kov, H., V. I v a n o v, G. Gad zh e v, K. G a n e v. Assessment of the Future Climate over Southeast EuropeBased on CMIP5 Ensemble of Climate Indices. Part One: Concept and Methods. - In: Proc. of 1st International Conference on ENVIROnmental Protection and Disaster RISKs, 2-4 October 2020, Sofia, Bulgaria (in Press).

8. Che r ve n k o v, H., V. I v a n o v, G. Gad z h e v, K. G a n e v. Assessment of the Future Climate over Southeast EuropeBased on CMIP5 Ensemble of Climate Indices. Part Two: Results and Discussion. - In: Proc. of 1st International Conference on ENVIROnmental Protection and Disaster RISKs, 2-4 October 2020, Sofia, Bulgaria (in Press).

9. Co nover, W. J. Practical Nonparametric Statistics. - In: Wiley Series in Probability and Mathematical Statistics: Applied Probability and Statistics. Vol. 350. Wiley, 1999, p. 336.

10. Fe r n a n d e s, R., S. G. Le b l a n c. Parametric (Modified Least Squares) and Non-Parametric (Theil-Sen) Linear Regressions for Predicting Biophysical Parameters in the Presence of Measurement Errors. - Remote Sensing of Environment, Vol. 95, 2005, No 3, pp. 303-316. DOI:10.1016/j.rse.2005.01.005.

11. Jeneinova, K., S. Kohnova, M. Sabo. Detecting Trends in the Annual Maximum Discharges in the Vah River Basin. - Slovakia, Acta Silv. Lign. Hung., Vol. 10, 2014, No 2, pp. 133-144. DOI: 10.2478/aslh-2014-0010.

12. K e n d a 1 1, M. G. A New Measure of Rank Correlation. - Biometrika, Vol. 30, 1938, pp. 81-93.

13. M a n n, H. B. Nonparametric Tests against Trend. - Econometrica, Vol. 13, 1945, pp. 245-259.

14. Meals, D. W., J. S pooner, S. A. Dressing, J. B. Harcum. Statistical Analysis for Monotonic Trends. - Tech Notes, Vol. 6, November 2011. Developed for U.S. EPA by Tetra Tech, Inc., Fairfax, VA. 23 p.

https://www.epa.gov/polluted-runoff-nonpoint-source-pollution/ sourcepollution/nonpoint-source-monitoring-technical-notes

15. Moss, R. H., et al. The Next Generation of Scenarios for Climate Change Research and Assessment. - Nature, Vol. 463, 2010, No 7282, pp. 747-756. DOI:10.1038/nature08823.

16. Pre s s, W. H., B. P. F 1 a n n e r y, S. A. T e u k o l s k y, W. T. V e t t e r 1 i n g. Numerical Recipes: The Art of Scientific Computing. 1986, pp. 213-214.

17. S e n, P. K. Estimates of the Regression Coefficient Based on Kendall's Tau. - Journal of the American Statistical Association, Vol. 63, 1968, pp. 1379-1389.

18. Sillmann, J., E. Roeckner. Climatic Change. Vol. 86, 2008, pp. 83-104. 10.1007/s10584-007-9308-6.

19. S i $11 \mathrm{~m}$ a n n, J., V. V. K h a r i n, F. W. Z w i e r s, X. Z h a n g, D. B r o n a u g h. Climate Extremes Indices in the CMIP5 Multimodel Ensemble. Part 2. Future Climate Projections. - J. Geophys. Res. Atmos., Vol. 118, 2013, pp. 2473-2493. DOI: 10.1002/jgrd.50188. 
20. S t o r c h, H., F. Z w i ers. Regression in Statistical Analysis in Climate Research. - Cambridge, Cambridge University Press, 1999, pp. 145-170. 10.1017/CBO9780511612336.009.

21. T h e i 1, H. A Rank-Invariant Method of Linear and Polynomial Regression Analysis. I, II, III. In: Nederl. Akad. Wetensch. Proc., Vol. 53, 1950. Part I: pp. 386-392, Part II: pp. 521-525, Part III: pp. 1397-1412.

22. T a y 1 o r, K. E., R. J. S t o u f f e r, G. A. M e e h 1. An Overview of CMIP5 and the Experiment Design. - Bull. Amer. Meteor. Soc., Vol. 93, 2012, pp. 485-498. DOI:10.1175/BAMS-D-1100094.1.

23. W i l c o x, R. R. 10.2 Theil-Sen Estimator. Introduction to Robust Estimation and Hypothesis Testing. Academic Press, 2005, pp. 423-427. ISBN 978-0-12-751542-7.

24. WMO (2000): Detecting Trend and Other Changes in Hydrological Data. WCDMP-45, WMO/TD 1013.

25. Y u e, S., Z. W. Kund zew i c z, L. W a n g. Detection of Changes. - Changes in Flood Risk in Europe, IAHS Special Publication, Vol. 10, 2012, Chapter 2, pp. 11-26.

26. Zhao, G., G. Hoermann, N. Fohrer et al. Streamflow Trends and Climate Variability Impacts in Poyang Lake Basin. - China, Water Resour. Manage, Vol. 24, 2010, 689. DOI:10.1007/s11269-009-9465-7.

Received: 15.09.2020; Second Version: 21.10.2020; Accepted: 23.10.2020 